\title{
RECENT ADVANCES IN POLARIZED ELECTRON SOURCES*
}

\author{
Charles K. Sinclair ${ }^{+}$, Jefferson Laboratory, Newport News, VA
}

\section{Abstract}

Current experimental physics programs at a number of electron accelerator laboratories worldwide require the delivery of high average current highly polarized electron beams for long periods of time. The polarized electrons are produced by near bandgap photoemission from certain semiconductor photocathodes. We observe the quantum efficiency of these cathodes to be inversely related to the total charge they have delivered. Recent developments in ultrahigh vacuum technology, electron trajectory control, photocathode preparation, and lasers have led to operationally reliable delivery of many hundreds of coulombs of polarized electrons, at rates as high as 8 coulombs/day. Currently, our photocathode operational lifetime is almost completely dominated by ion backbombardment. Further gains in the high average current lifetime of these cathodes may be expected, which will allow photoemission electron guns to be used for accelerator applications other than polarized sources.

\section{INTRODUCTION}

During most of the past decade, polarized electron source development efforts have concentrated on developing cathodes to provide electron beam polarization above the $\sim 40 \%$ available from GaAs; on reducing the "charge limit" effect; and on establishing ultrahigh vacuum conditions in the polarized electron guns sufficient to provide reasonable photocathode operational lifetimes. The charge limit effect is a current dependent reduction in the effective cathode quantum efficiency, caused by space charge accumulation at the surface of the semiconductor cathode. This effect is important in injectors for low duty factor accelerators. Most of the ultrahigh vacuum work centered on the development of various load-lock schemes, which allowed photocathodes to be activated outside the high voltage section of the gun, and installed in the electron gun without the need for a vacuum bakeout. These developments have led to a number of polarized electron injectors which deliver beam polarizations of about $80 \%$ from cathodes with initial quantum efficiencies in the 0.1 to $0.3 \%$ range at the instantaneous currents used. Cathode "dark" lifetimes of 1000 hours or greater are routinely obtained in most laboratories, while at average beam currents of about 20 $\mu \mathrm{A}$, the cathode lifetimes are typically an order of magnitude shorter. To date, all cathodes which deliver very high electron polarization have the unfortunate side

"Work supported by US DOE under contract DE-AC05-84ER40150 + Email: sinclair@jlab.org effect of showing significant polarization correlated beam intensity and position changes, complicating their use in physics experiments.

With the advent of $\mathrm{CW}$ electron accelerators, the demand for much higher average beam currents and much higher total charge delivery per experiment arose. In addition, the need to do experiments with the highest practical beam polarization requires that the polarization correlated systematic effects be either eliminated or carefully controlled. The means to control these effects are under active study at the present time, and will not be discussed further here. The polarized source technologies in place as of early 1997 were unable to meet the high average current and high total charge requirements while providing acceptable photocathode operational lifetimes. Over the past two years, several developments have taken place which now allow the delivery of hundreds of coulombs of highly polarized electrons, at rates up to 8 coulombs/day.

The term "operational lifetime" is used to describe the length of time that the cathode can be used to deliver beam meeting established requirements, rather than a cathode quantum efficiency decay constant. This operational lifetime is the convolution of many system parameters and physical effects, such as the initial quantum efficiency and the available laser power; the total pressure and residual gas composition of the static vacuum in the gun; vacuum degradation during operation with beam; electron losses between the photocathode and the experimental target; the useful photocathode area, etc.

\section{VACUUM ISSUES}

There are two classes of vacuum issues associated with photoemission electron gun operation. The first of these is degradation of the cathode quantum efficiency by chemical poisoning of its surface. The photocathode is prepared, or "activated", by exposure of the clean semiconductor surface to monolayer quantities of cesium and an oxidant. The dark lifetime of the finished cathode is strongly affected by exposure to tiny quantities of chemically active gases, such as water, oxygen, and carbon dioxide. It is generally suspected that additional oxidation of the cathode is involved in this degradation, but convincing evidence is difficult to establish rigorously, as the quantities of gases involved are exceptionally small [1]. Experience at many laboratories has shown that it is possible to prepare good quantum efficiency cathodes in a polarized gun with dark lifetimes of one thousand to several thousand hours. This indicates that to the extent that chemical poisoning of the cathode is responsible for reduced cathode operating life, it is caused by gases released by electron-stimulated desorption. 
The electrons responsible for this desorption may arise either from field emission from the gun electrode structures, or electron beam losses in areas which can communicate, vacuum-wise, with the cathode. Generally speaking, it is practical to eliminate field emission as a source of cathode lifetime degradation [2]. Note that chemical poisoning should degrade the cathode quantum efficiency uniformly over its entire area.

The second vacuum related issue is ion backbombardment of the cathode. Depending on the ion mass and energy, the damage to the cathode may range from generation of defects in the cathode semiconductor to sputtering of the oxidized cesium surface layer. While ion damage may depend somewhat on the ion mass, it does not depend on the chemical nature of the ion. Thus the total system pressure, rather than the residual gas composition, is the more relevant parameter in quantifying this damage mechanism.

In many polarized electron guns, the photocathode is not uniformly illuminated. In these cases, ion backbombardment would be expected to affect only the area of the cathode between the illuminated spot on the cathode and the electrostatic center of the cathode. In the case of a fully illuminated cathode, ion backbombardment would preferentially damage the region around the electrostatic center of the cathode. Thus, by comparing maps of the cathode quantum efficiency before and after operation with beam, one should be able to discriminate between chemical poisoning and ion backbombardment problems. It is worth noting that to the extent that the cathode lifetime is limited by ion backbombardment, it is far more meaningful to express the lifetime in terms of the delivered charge per. unit illuminated area of the cathode, rather than clock hours or calendar days.

Evidence of cathode degradation at the illuminated site was first reported by groups at Mainz and Bates [3], and the Mainz group first reported the ion backbombardment interpretation of this [4]. An example of a quantum efficiency map showing clear evidence of ion backbombardment damage is shown in figure 1 , taken from the $100 \mathrm{kV}$ Jefferson Lab polarized gun.

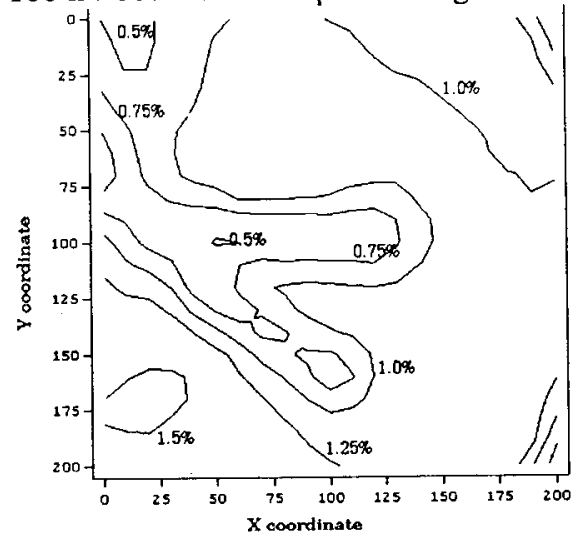

Figure 2. A quantum efficiency map of a cathode damaged by ion backbombardment
Here, a small diameter $(-250 \mu \mathrm{m})$ laser beam illuminated the cathode in the two indicated locations. The damage region between the illuminated spots and the electrostatic center of the cathode is clear. Some fraction of this damage can be repaired by heat cleaning and re-activating the cathode. Ion backbombardment damage is an important effect in polarized electron guns operating at MAMI and Bates, and is the dominant cathode damage mechanism at Jefferson Lab. In the present Jefferson Lab polarized gun, a $1 / \mathrm{e}$ reduction of the quantum efficiency in the ion damaged area corresponds to the delivery of $\sim 10000$ coulombs $/ \mathrm{cm}^{2}$.

There is some evidence that cathodes which produce the highest beam polarization also have better operational lifetimes than ordinary GaAs cathodes. The reason for this may be that all high polarization cathodes developed to date are very thin - typically about $100 \mathrm{~nm}$. Ions of the highest energy, which might be expected to do the greatest damage to the cathode, pass through this thin layer and deposit the majority of their energy in the underlying substrate. For example, hydrogen ions with energies above $\sim 15 \mathrm{keV}$ will pass through a $100 \mathrm{~nm}$ layer of GaAs. It also appears true that the quantum efficiency loss caused by backbombardment with lower energy ions may be recovered more readily by heat cleaning and reactivation.

The only beam produced ions which have much likelihood of being accelerated back to the cathode must originate in the cathode-anode gap. Most polarized guns constructed to date have not had particularly good pumping speed in the cathode-anode gap region. At Jefferson Lab, we have constructed a gun in which the cathode has been extended into a chamber containing a massive array of NEG pumps [5]. When fully activated, these pumps provide a pumping speed in the cathode chamber of over $4000 \mathrm{~V} / \mathrm{sec}$ for $\mathrm{H}_{2}$ and $\sim 1700 \mathrm{l} / \mathrm{sec}$ for CO.

In its first test, this gun reached a base pressure, measured by an extractor gauge located in the chamber downstream of the anode aperture, of $\sim 2 \times 10^{-12}$ torr. The sensitivity limit of this gauge is claimed by the manufacturer to be $\sim 1 \times 10^{-12}$ torr. RGA scans indicate that the pressure in the gun is higher when the gauge filament is powered. Thus we believe that we have achieved a very excellent static vacuum in this gun. This gun has been in service for 14 months, and has consistently provided the excellent cathode charge delivery reported above. This gun is shown in figure 2.

Gases may reach the cathode-anode gap region from higher pressure regions downstream of the gun. In the Jefferson Lab injector the pressure in these downstream areas gradually increases to about $10^{-9}$ torr several meters from the cathode. These regions are isolated by sputter ion pumps and a NEG based differential pump module. In an effort to further decrease the pressure in the cathodeanode gap, we have sputter coated the inner wall of the 


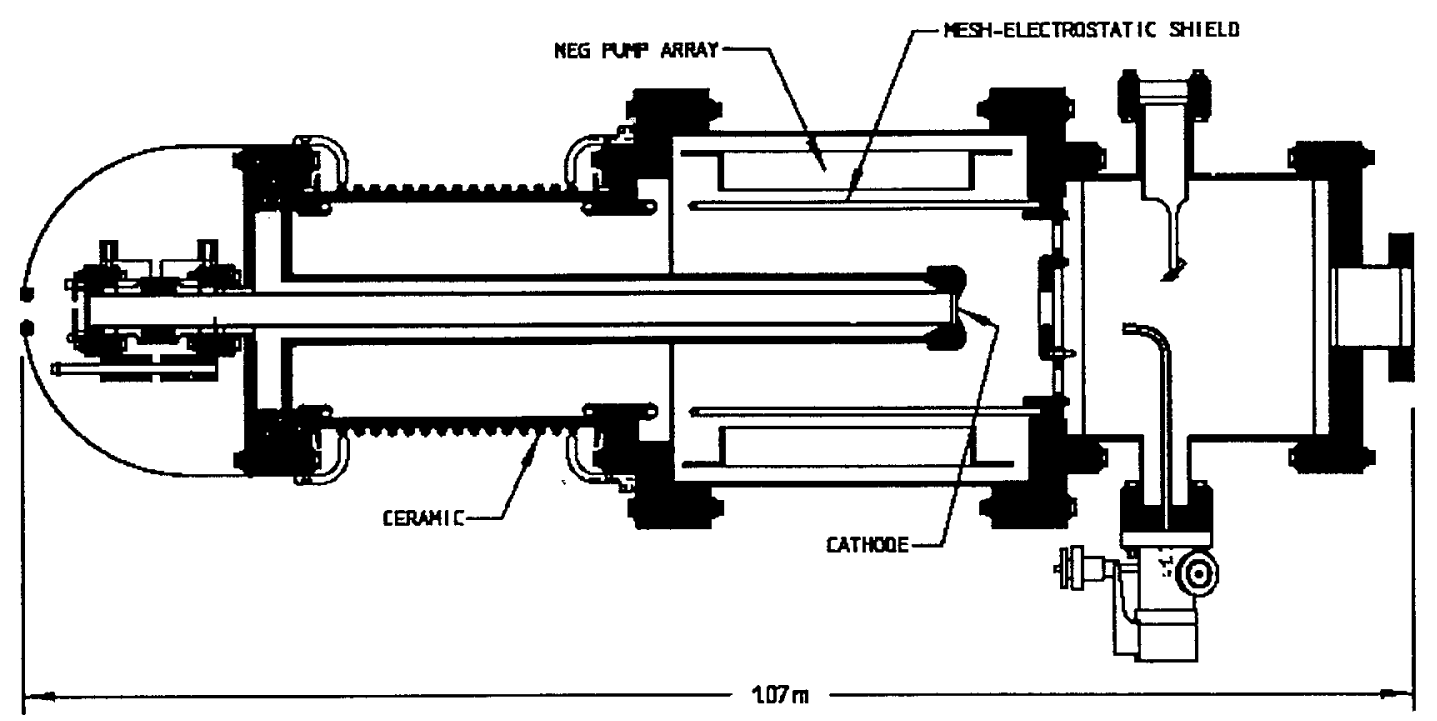

Figure 2. Schematic view of the Jefferson Lab polarized gun.

vacuum tube downstream of the gun with a $\mathrm{Ti} / \mathrm{Zr}$ getter, following the method of Benvenuti et al. [6]. This coated beam tube provides three distinct vacuum advantages. It provides a high pumping speed for chemically active gases; it is a diffusion barrier to gases diffusing from or permeating through the beam tube wall; and it is expected to have dramatically lower electron stimulated desorption [7]. We have found Benvenuti's method straightforward to reproduce, and a gun incorporating a coated beam tube is presently in test.

\section{ELECTRON TRAJECTORY CONTROL}

During early use of the Jefferson Lab polarized source, good operational lifetimes were obtained with average currents in the range of $10-20 \mu \mathrm{A}$. At currents of 100 $150 \mu \mathrm{A}$, lifetimes were substantially shorter. At these higher currents, we observed a significant rise in the vacuum pressure in the beamline following the polarized gun, and sensitive Geiger tubes placed along this beamline showed an elevated counting rate with beam on. These are clear indications of electron beam striking the beamline vacuum chambers. This was initially difficult for us to understand, since the 12.8 $\mathrm{mm}$ diameter cathode was illuminated by a $0.2 \mathrm{~mm}$ diameter laser spot placed close to the cathode center. The beam spot observed on all downstream viewscreens was very small, as anticipated, and modelling results indicated that the beam originating from the directly illuminated area of the cathode should be far from all vacuum system walls. We concluded that a small number of beam electrons must be originating from large radius areas of the cathode. These large radius electrons follow extreme trajectories, ultimately striking the beam tube walls. The extreme trajectories were the result of small transverse fields at the edge of the cathode; short focal lengths of some beamline elements; and field aberrations in the solenoids. Modelling with PARMELA, using the actual fields of these elements, demonstrated that these extreme trajectories struck the vacuum walls [8].

Initially, we thought that the electrons originating at large radius were due to reflections from the window admitting laser light into the gun. Replacement of this window with an AR coated version produced no change in the beam loss, where a factor of eight reduction would have been expected were these reflections the source of the problem. We presently believe that the large radius electrons originate from photoemission caused by recombination light in the semiconductor cathode itself. This recombination light, which is close to the bandgap energy of the semiconductor, is weakly absorbed and thus can reach the large radius areas of the cathode wafer.

To reduce the number of electrons originating at large radius on the cathode, we prepared cathodes with the quantum efficiency deadened at large radius. Initially we reduced the quantum efficiency by masking the large radius area during atomic hydrogen cleaning. More recently, we have grown a thick anodic oxide on the large radius region of the cathode. The anodized region has zero quantum efficiency at the illumination wavelength. The previously observed pressure rise and Geiger tube counting rate were dramatically reduced in the first test with a cathode deadened at large radius. Unfortunately, this improvement was introduced at the same time as the massive NEG pump array, and we do not know the relative contributions of these two changes to the cathode lifetime improvement.

In the gun system now in test, noted above, we have changed to a horizontal gun mounting, eliminating a 90 degree bend magnet which had both short focal lengths and a relatively small gap. We have increased the bore of the downstream vacuum tube as well as coating it with NEG material. The solenoid closest to 
the gun now has an increased bore, reducing its aberrations. Finally, we have altered the shape of the cathode electrode, and increased the anode aperture, reducing the small transverse fields in the cathode area. We anticipate that each of these changes will further improve the operational lifetime of the cathode.

\section{REDUCTION OF ELECTRON LOSSES BETWEEN PHOTOCATHODE AND EXPERIMENTAL TARGET}

It is clear that even with the improvements obtained by providing exceptional vacuum conditions, it is desirable to produce only electrons which can be transported to the ultimate user. For accelerators requiring single or widely spaced bunches, and where high accelerator energy and/or damping rings provide adequate beam quality, subharmonic bunching provides an effective solution, as at the SLC. For CW or high duty factor accelerators, this technique is not applicable. Furthermore, in these latter machines, it is normal to limit the emittance growth associated with bunching by RF chopping the beam before the buncher. Typically, $65 \%$ to $85 \%$ of the beam leaving the cathode is lost in such chopping systems.

To avoid the chopping losses, it is necessary to produce the electrons already bunched at the cathode, and/or to extend the fraction of the RF cycle over which the buncher can compress the beam without degrading the beam quality. Both schemes are now employed at CW machines.

At MAMI, two buncher cavities are used, the first operating at the fundamental frequency and the second at the second harmonic. With the two cavities separated by the correct distance, the bunching field is effectively linearized over about $50 \%$ of the RF cycle. Using this technique, the MAMI injector increased the acceptance of their chopper-buncher system from $-15 \%$ to $\sim 50 \%$ [9].

At Jefferson Lab, we have developed an RF gainswitched diode laser oscillator-amplifier system [10]. Variations of this laser have been demonstrated to operate reliably between $125 \mathrm{MHz}$ and $3 \mathrm{GHz}$. At Jefferson Lab, three such lasers, each operating on the third subharmonic of the $1497 \mathrm{MHz}$ fundamental accelerator frequency, produce three interleaved bunch trains, which are delivered to three independent experimental halls with the aid of third subharmonic RF separators. Longitudinally polarized electron beams have been delivered to all three experimental halls simultaneously with this scheme. MAMI has adopted this laser system as well, and in conjunction with its two frequency bunching system, now delivers $\sim 95 \%$ of a $20 \mu \mathrm{A} \mathrm{CW}$ beam from its photocathode to the experimental target. At Jefferson Lab, where the polarized beam current to a single experimental hall has been as high as $110 \mu \mathrm{A} \mathrm{CW}$, a pre-buncher is used ahead of the chopper system to optimize the transmission. $75 \%$ transmission from cathode to target has been achieved to date at this high current, with higher transmission obtained at lower current. Future experiments planned at Jefferson Lab will require six times the present peak microbunch current. Additional effort will be necessary to accomplish this.

It is worth noting that with RF structure present at the cathode, standard BPMs can be used in the low energy region of the injector. Such BPMs have been implemented at Jefferson Lab, and are very useful for beam setup.

\section{IMPROVING CATHODE PREPARATION}

All else being equal, a high initial quantum efficiency will deliver the longest cathode operational lifetime. For the highest quantum efficiency, the semiconductor surface should be clean on an atomic scale prior to the activation with cesium and oxidation. Some surface contaminants, such as carbon and some oxides, are difficult or impossible to remove by conventional heat cleaning. All cathodes developed to date to provide high beam polarization are too thin $(-100 \mathrm{~nm})$ to tolerate wet chemical cleaning. We have developed a cathode cleaning technique based on exposure of the cathode surface to atomic hydrogen, which is well known to produce atomically clean surfaces on many semiconductors. The atomic hydrogen is produced in a small RF discharge chamber [11]. The dangling bonds at the surface of atomically clean GaAs are saturated by atomic hydrogen exposure, so the surface is effectively passivated and can be transferred through air into an electron gun structure [12]. Whether this is true for other semiconductors remains to be demonstrated. Using atomic hydrogen cleaning, we have consistently produced high quantum yield photocathodes on both strained and unstrained GaAs surfaces.

The excellent results we have had using atomic hydrogen cleaning have led us to examine the importance of the cathode dopant density on the maximum quantum efficiency. It has been shown that a high dopant density, at least near the cathode surface, is important to reduce the charge limit effect [13]. However, since a high dopant density is responsible for electron depolarization in the cathode in several theoretical models, it may be possible to improve the polarization by lowering the dopant density in applications where the charge limit is not important. To determine the importance of the dopant density, we procured a set of six different epitaxial GaAs wafers of varying thickness and dopant density. The GaAs layers were grown on a thick layer of GaAlAs, with the aluminum fraction chosen so that for wavelengths 
longer than about $670 \mathrm{~nm}$, all photoemission was from the thin GaAs layer only.

The quantum efficiency of one of these thin GaAs samples is shown in figure 3 . This sample was 0.27 $\mu \mathrm{m}$ thick, and was p-doped at $5.8 \times 10^{17} \mathrm{~cm}^{-2}$.

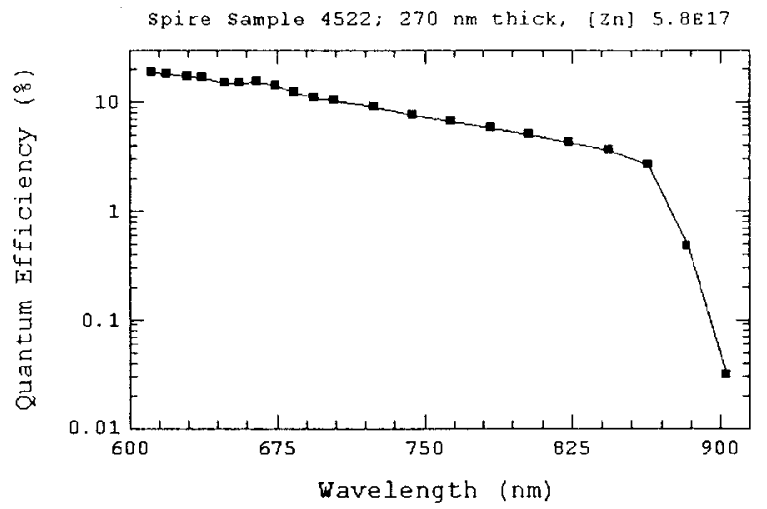

Figure 3. Quantum efficiency versus wavelength for a thin, lightly doped GaAs sample.

A two parameter fit to the 700 to $850 \mathrm{~nm}$ wavelength region, using the known absorption coefficient and its variation with photon energy, yields an electron surface escape probability of 0.26 [14]. This escape probability is considerably higher than both modelled and measured values for the surface escape probability reported in the literature [15]. We do not know if this excellent esape probability is a result only of the atomic hydrogen cleaning process. However, we have obtained escape probabilities comparably higher than those reported in refs. 15 from three other thin, lightly doped samples. Further measurements on these samples are planned to determine the effect of dopant density on polarization.

\section{SUMMARY}

Prior to 1998, polarized electron injectors were delivering several tens of coulombs to experimental programs during an entire calendar year. The future experimental program planned at Jefferson Laboratory requires the delivery of many hundreds of coulombs annually from high polarization cathodes. Both the Bates Lab and Jefferson Lab demonstrated during 1998 the delivery of several hundred coulombs to their experimental programs during two to three month periods from bulk and thin GaAs photocathodes. At Jefferson Lab, excellent operational lifetime at high average current from high polarization cathodes has recently been demonstrated. These accomplishments have been made without the use of complex load-locked electron guns, although well designed load-locked guns offer clear operational versatility which is difficult to achieve otherwise.

Ion backbombardment of the cathode is presently the dominant limiting factor in the operational lifetime of photoemission cathodes for polarized electron delivery. State-of-the-art ultrahigh vacuum technology and the elimination of electron stimulated desorption by careful control of extreme electron trajectories in the polarized gun and its associated beamline can reliably provide very long cathode operational lifetimes. Methods to maximize the fraction of the photoemitted electrons which reach the experimental target, such as RF gainswitched lasers and improved bunching schemes, are also very useful. At Jefferson Lab, 1/e quantum efficiency degradation due to ion backbombardment from the delivery of $\sim 10000$ coulombs $/ \mathrm{cm}^{2}$ has been demonstrated. With additional improvements in the operating vacuum in the cathode-anode gap of the electron gun, considerably greater charge delivery appears possible.

\section{ACKNOWLEDGMENTS}

The development of the Jefferson Laboratory polarized electron source has involved the work of many individuals. In particular, Phil Adderley, Tony Day, Bruce Dunham, John Hansknecht, Peter Hartmann, Reza Kazimi, Hongxiu Liu, Danny Machie, Matt Poelker, Scott Price, Paul Rutt, Bill Schneider, and Michael Steigerwald have all made major contributions to the work at Jefferson Lab. Manouchehr Farkondeh, Kurt Aulenbacher, and Jym Clendenin provided information on the polarized source work at the Bates Laboratory, MAMI, and SLAC, respectively. Khaled El Amrawi and Prof. Hani Elsayed-Ali, of Old Dominion University, participated in our initial development of the atomic hydrogen cleaning process.

\section{REFERENCES}

[1] H. Fischer et al.,Proceedings of the Workshop on Photocathodes for Polarized Electron Sources for Accelerators, SLAC Report 432 Rev, SLAC, Stanford, CA, 1994, p. 249

[2] At Jefferson Lab, we have obtained very good high voltage performance from electrodes made of Ti-6Al-4V alloy.

[3] C. K. Sinclair, in High Energy Spin Physics, AIP Conference Proceedings No 187, AIP, New York, p. 1412.

[4] K. Aulenbacher et al., Proceedings of the Workshop on Photocathodes for Polarized Electron Sources for Accelerators, SLAC Report 432 Rev. SLAC, Stanford, CA 1994, p.1.

[5] NEG pumps are commercially supplied by SAES Getters.

[6] C. Benvenuti et al., J. Vac. Sci. Technol. A16, 148 (1998).

[7] J. J. Welch and C. K. Sinclair, Proceedings of the 1986 Linear Accelerator Conference, SLAC Report 303, SLAC, Stanford, CA, p. 87.

[8] B. Dunham, private communication.

[9] V. T. Shvedunov et al., EPAC96, p. 1556.

[10] M. Poelker, Appl. Phys. Lett. 67, 2762 (1995)

[11] M. Poelker et al., Nucl. Instr. Meth. A364, 58 (1995).

[12] Y. Okada and J. S. Harris, J. Vac. Sci. Technol. B14, 1725 (1996).

[13] K. Togawa et al,, Nucl. Instr. Meth. A414, 431 (1998)

[14] J. S. Blakemore, J. Appl. Phys. 53, R123 (1982).

[15] D. G. Fischer et al., J. Appl. Phys. 43, 3815 (1972); G. Vegara et al., J. Vac. Sci. Technol. A8, 3676 (1990). 